nieurs civils ef Perrin, directeur de l'Ecole Professiomnelle, ont éte adjoints à la Commission pour l'aider à prendre ses mesures.

Les résultats obtenus devant elle marquent $m$ grand pas fait en avant dans le difficile problème du transport de la force a grande distance, et ils ont dépassé de beaucoup ceux que M. Marcel Deprez avait annoncés. A un moment donné, on a reçu jusqu'à 7 chevaux à Grenoble, avec un rendement supérieur à $60 \%$. Ce résultat est unique au monde.

Notre journal aura une durée éphémère, mais il présente cette étomnante originalité d'ètre tiré sur une presse à imprimer qui prend sa force à 14 kilomètres de la ville, et la reçoit au moyen de deux fils qui ont 2 millimètres de diamètre. Ceux qui, trop peu avancés dans l'étude des sciences, ne peuvent pas se rendre compte des merveilleuses découvertes de $M$. Marcel Deprez, seraient au moins frappés de ce fait et admireront le grand savant qui la réalisé.

Pour qu'il reste un souvenir de tous ceux qui, de près ou de loin, ont touché à cette magnifique expérience, nous donnons la composition du Conseil municipal de la ville de Grenoble, au moment où nous écrivons :

MM. Rey Edouard, maire ; Eymard, premier adjoint :DurandSavoyat, deuxième adjoint; Germain, troisième adjoint ; Dantart, Bergès, Bernard, Bertrand, Calvat Ernest, Cavalis, Cauley, Charlon, Collet, Durand Léon, Gauthier Abel, Giraud, Guigonnet. Jar, Marquian, Pellet, Poulat, Porteret, Refait, Tarillon, Testoud, Laurat, Robert, conseillers.

En terminant, qu'il me soit permis de dire un mot de la presse grenobloise : MM. Maisonville, rédacteur en chef de l'Impartial des Alpes, Mascet, rédacteur en chef du Réveil du Dauphiné, Claude, rédacteur en chef du Républicain de l'Isère, Roux, correspondant du Lyon Républicain, Gaillard, de l'Impartial des Alpes, Dumas, du Réveil du Dauphiné, ont laissé de côté toute question de clocher, et nous ont été de précieux intermédiaires auprès du public. Qu'ils en acceptent ici tous nos remerciements. Jules SARcra.

\section{AVENIR}

L'avenir n'est à personne, et il n'est pas possible, au début d'une npplication aussi importante, d'en cléfinir d'avance les résultats, mais il est très permis, d'après la rapidité de sa marche passée, la sûreté des résultats acquis, d'en prévoir au moins le développement prochain.

Le transport de la force n'a que quelques années d'existence. Au Congrès d'électricité en 1881, des contradictions, des doutes entourèrent l'exposé des doctrines de M. Marcel Deprez, alors appuyées seulement sur des essais de laboratoire. Deux années ne sont pas écoulées, voici les machines en marche et l'application pratique réalisée.

Les conséquences de cette découverte sont connues de tous. L'accroissement immense de richesses qui résultera de la récolte et de l'apport dans les villes d'une quantité illimitée de forces jusqu'ici perdues, est un fait évident; les heureuses modifications sociales, l'agrandissement de l'initiative individuelle qui sortiront de la distribution de la force ont été souvent signalés. Spécialement à Grenoble, dans ce pays de forces naturelles, d'industrie active et d'intelligence pratique, il est superflu d'insister sur ces choses.

Ce qu'il faut dire, c'est qu'il n'y a plus d'incertitudes à conserver sur cet avenir. $\mathrm{Si}$, après les expériences de laboratoire, même après les expériences contrôlées par de si hautes autorités, faites au chemin de fer du Nord, si, disons-nous, après ces faits un murmure de contradictions pouvait encore s'élever, il n'en est plus de mème aujourd'hui ; l'application de Grenoble a été pratique à tous les points de vue : chutes d'can, ligne télégraphique, valeur de la force reçe ; elle a été contròlée sur tous les points ; elle aura duré un mois avec des marches journalières; le doute doit cesser. l'avenir est immense et il est assuré.

Frank Géraldi, Jules Sarcia.

\title{
L'Enseignement Technique à l'Exposition de Grenoble
}

Les expositions des diverses classes de l'Enseignement Technique, à Grenoble, occupent, avec la librairie technique, la totalité du développement des galeries du premier étage du grand Palais de la Houille blanche.

La classe 15 "Grandes Ecoles de l'Etat ", parmi lesquelles nous regrettons malheureusement la non-participation de l'Ecole Polytechnique, comporte les expositions de l'Ecole Centrale des Arts et Manufactures (photographies de laboratoires), celle de l'Ecole Nationale des Ponts et Chaussées (programme de cours et gravures anciennes représentant les célébrités ayant appartenu au corps des ingénieurs des Ponts et Chaussées), et encore celle de l'Ecole Nationale Supérieure des Mines (photographies de laboratoires, cours d'électrotechnique et ouvrage sur les manipulations). Bien plus largement représentés sont le Conservatoire National des Arts et Métiers et l'Ecole Supérieure des Postes et Télégraphes.

Le premier établissement offre un certain nombre d'appareils de démonstration, suivant les conceptions matérielles chères aux professeurs anglais (appareils mettant en évidence l'influence des champs alternatifs polyphasés sur des masses métalliques non magnétiques (moteurs asynchrones) et sur une aiguille aimantée (moteurs synchrones); à citer également un transformateur (lampe à trois électrodes pour très haute fréquence, 300.000 périodes par seconde) ; un électroaimant excité par une simple pile de lampe de poche, des appareils de démonstration, ampèremètre et électrodynamomètre ; enfin, de belles photographies et de suggestives solutions graphiques de problèmes d'électrotechnique élémentaire.

L'Ecole Supérieure des Postes et Télégraphes expose différents modèles d'appareils téléphoniques, entre autres la boîte à cigares dont le précurseur français, Boursault, avait fait, dès 1854 , un appareil téléphonique. En outre de publications sur des essais de téléphonométrie, à signaler une très curieuse maquette de lignes télégraphiques et téléphoniques, due à $\mathrm{M}$. Pierrer, fonctionnaire de l'Administration.

La classe 16 "Instituts techniques d'Enseignement supérieur, Ecoles supérieures diverses ", est, dans son ensemble, beaucoup plus intéressante.
L'Ecole Supérieure d'Electricité de Paris expose des graphiques fort importants relatifs à ses mouvements d'élèves, puis les éléments constituants d'une ligne artificielle représentant un transport d'énergie à très haute tension et à très grande distance. L'installation comporte, en plus de la ligne proprement dite, une station génératrice, des appareils récepteurs et un dispositif approprié pour les mesures. La station génératrice qui comprend essentiellement un alternateur diphasé, permet d'alimenter la ligne à tension constante, mais à phase variable. La ligne est, du reste, établie d'une longueur équivalente à peu près égale à un quart d'onde. Ces machines spéciales, étudiées par les ateliers de "Lyon-Dauphiné ", à Lyon, sont particulièrement intéressantes et méritent un examen approfondi. Regrettons cependant que l'installation n'ait pu être mise en marche effective.

L'Institut Electrotechnique de Nancy montre d'intéressants graphiques et de beaux laboratoires de recherches et d'enseignement, notamment son laboratoire de recherches hydrauliques, fondé en 1922. La station de jaugeage par moulinets hydrométriques est particulièrement séduisante. En outre, les traditionnels projets d'élèves et comptes rendus d'essais.

L'Institut Electromécanique de Lille, tout récent, destiné à remplacer, incomplètement du reste, l'ancien Institut Electrotechnique détruit par un incendie durant la guerre, n'expose que des cours, du reste fort remarquables, de ses divers professeurs.

L'Office National des Recherches scientifiques et industrielles et des inventions, montre l'appareil du clocteur Panis pour la respiration artificielle, appareil éminemment utile et humanitaire.

L'Institut Technique de Normandie (Caen) et l'Institul Polytechnique de l'Ouest (Nantes), offrent aux visiteurs des séries de travaux et de graphiques intéressants, mais un peu maigres. Citons encore l'Institut Electrotechnique de Toulouse, dont la rèputation, notamment au point de vue hydraulique, où son directeur est passé maître, n'est plus à faire (station de tarage des moulinets hydrométriques et laboratoire d'hydraulique de Banlève); une très artistique affiche illustrée complète cette exposition. 
L'Anstitut d'Electrochimie et d'Electrométallurgie de Grenoble, sous la direction du très distingué professeur Flusin, expose des plans fort importants de ses futures installations, et des produits électrométallurgiques déjà fabriqués dans ses laboratoires. On sait que, créćs par l'Institut Polytechnique de I'Université de Grenoble, les laboratoires et enseignements d'électrochimie et d'électromélallurgie de cette Université, en raison même du développement qu'ils ont acquis, vont être concentrés en même temps que très développés dans un Institut spécial autonome, dont une grande partie des bâtiments est déjà édifiée.

A citer encore le groupe des Ecoles supérieures placées sous le patronage de la Chambre de Commerce de Lyon, qui expose des tableaux statistiques, des photographies et des exemplaires de cours et des bulletins des Associations d'anciens élèves. Ecole Centrale de Lyon, Fcole de Chimie industrielle et Ecole Française de Tannerie, Ecole Supérieure de Commerce et de Tissage, Cours coloniaux de Lyon.

Deux écoles spécialistes en matière électrotechnique, l'Ecole Bréguet, à Paris, et l'Ecole d'Electricité industrielle Charliat, de paris également, montrent aux visiteurs des photographies d'installations, des graphiques de mouvements scolaires, et enfin des pjèces d'appareillage et installations électriques exécutées par Jeurs élèves.

L'Institut Polytechnique de Grenoble se devait, en raison de sa situation régionale et aussi de sa spécialisation évidente pour tous les renseignements et recherches relatifs à la houille blanche de manifester, par une exposition réellement hors de pair, son importance à ce double point de vue. Qu'on nous excuse, bien que nous ne disions que la vérité, de l'affirmer avec quelque amour paternel. Dans l'occurrence, l'aveuglement du père hibou du bon Lafontaine ne sera peut-être pas tout à fait absent de notre exposé.

Les services d'enseignement de l'Institut de Grenoble qui comportent, comme on le sait, plusieurs sections destinées à former diverses catégories d'ingénieurs, exposent un panneau général d'ensemble comportant des vues de toutes les installations, des graphicfues représentatifs du mouvement scolaire qui, comme on le sait, est considérable, puisque l'effectif se maintient, après oscillations de la guerre et de l'après-guerre, à 800 élèves environ. En outre, une petite bibliothèque renferme une centaine d'ouvrages relatifs à la production, à la distribution et aux applications de l'électricité, à l'hydraulique, à la mécanique et à la papeterie. Tous ces ouvrages sont les cuvres des divers professeurs et d'anciens élèves, aujourd'hui ingénieurs, de l'Institut. A signaler également les expositions particulières des laboratoires d'essais industriels et de recherches scientifiques, celles notamment du laboratoire des essais électriques et du laboratoire des essais physiques et mécaniques des métaux, chaux et ciments. Des panneaux spéciaux de photographies et de schémas pour la papeterie, la mécanique, etc., encadrent le tableau principal.

Enfin, dans un stand particulier, le Bureau des Essais électriques de l'Institut a installé un véritable laboratoire permettant l'essai immédiat de tous appareils industriels de mesures électriques à courants alternatifs, monophasés et triphasés, de 0 à 400 ampères et de 0 à 600 volts. Sur un banc d'essai sont montés des compteurs de divers types et en particulier d'énergie réactive ; des clichés lumineux avec diagrammes montrent quels montages corrects sont à adopter et quels montages incorrects sont à rejeter. A signaler également l'exposition spéciale de la section du Service des Essais, qui s'occupe des réceptions d'usines hydrauliques, et qui a été appelée à fonctionmer bien souvent, non pas seulement dans la région du Dauphiné et de la Savoie, mais aussi jusqu'à Dijon, jusqu'à Clermont-Ferrand et même dans les Pyrénées. Une carte des principales centrales hydroélectriques, de la région des Alpes, a été établie par le Service et fait une partie de l'exposition. A signaler également une véritable station de jaugeage du débit des canaux par moulinets hydrométriques. Une installation de pompes d'épreuve permet de comparer tous manomètres industriels aux manomètres étalons du Bureau d'essais. Le laboratoire des essais et recherches à haute tension expose également des graphiques et des photographies d'expériences effectuées à 450.000 volts.

A citer également dans le groupe 1, classe 1 (Services d'Etat), les expositions particulieres de trois sections de l'Institut : le Service spécial d'Essais hydrauliques, le Service des Essais électriques à haute tension, enfin le laboratoire d'Essais mécaniques et physicues des métaux, chaux et ciments. Ces trois Services travaillent en liaison, non seulement avec l'industrie régionale, mais aussi avec les Pouvoirs Publics, d'où leur place clans ce groupe.
Enfin, bien qu'il ne soit plus guère en cela question d'euseignement, ajoutons que l'Institut Polytechnique, ou plutôt l'Ecole Française de papeterie qui se constitue l'une des sections, a organisé, dans le groupe VIII, classe 31 "Au Temple du Bois ", une exposition rétrospective du papier fort intéressante. On y voit le premier modèle de la machine Robert d'Essonnes qui, après avoir créé une source de richesse incalculable, est mort dans la misère : une pile à maillets d'Auvergne, à fabriquer le papier, suivant un mode très primitif, etc...

Signalons, enfin, que l'exposition des classes 15 et 16 , du groupe V, a été complètement organisée par l'Institut Polytechnique de Grenoble, et en particulier par M. Lafont, ingénieur-chef du Service des Essais, secrétaire de la classe 16. C'est au même ingénieur que l'on doit l'étude et la mise en place des enseignes générales des classes 17 et 18 , en outre de toutes celles des classes 15 et 16 .

La classe 17 "Ecoles Nationales d'Arts et Métiers " donne une impression d'ordre et d'étude d'ensemble réellement impressionnante. Les deux Ecoles d'Aix-en-Provence et de Cluny, qui se recrutent surtout dans la région du Sud-Est, ont eu à tenir le drapeau du groupe des Ecoles Nationales. Nombreuses photographies d'ateliers de modelage, de fonderie, de métallurgie. L'importance des traitements thermiques et des essais physiques des métaux, et celle des études métallographiques, ont été hautement reconnues des directeurs de ces Ecoles.

Aix donne, en un tableau très clair, l'organisation d'un bureau de travaux, puis une documentation sur l'usinage en série et stadardisation. A citer même des machines construites dans les ateliers de l'Ecole, une bâche spirale de turbine "Francis ", un groupe électrogène à turbine "Banki ", avec dynamo à courant continu.

L'Ecole de Cluny expose une turbine "Pelton " attelée à une dynamo, des collecteurs, un tour parallèle, etc... puis des copies de dessins et des résultats d'essais.

L'Association des Anciens Elèves des Ecoles Nationales d'Arts et Métiers affirme sa puissance par une affiche très prenante, montrant la diffusion des ingénieurs sortis de ces Ecoles dans les différentes branches de l'activité industrielle. Nous sera-t-il permis cependant de remarquer que si ceux-ci, en grand nombre comme le proclame l'affiche "participent à l'aménagement de la houille blanche et à la distribution de l'énergie électrique "avec un succès que nous reconnaissons d'autant plus volontiers que nous sommes l'initiateur de l'admission des anciens élèves des Arts et Métiers dans les Instituts Universitaires français, cette influence indéniable est surtout due à ce fait qu'avec l'excellente éducation mécanique reçue dans les Ecoles d'Arts et Métiers, ces vaillants praticiens vont, en nombre de plus en plus grand, demander la spécialisation électrotechnique indispensable, soit dans les Instituts Electrotechniques, soit à l'Ecole Supérieure d'Electricité. A cela nul déshomneur. La complication de la carrière industrielle est telle que la spécialisation ne peut guère ètre assurée que par ces spécialistes.

Enfin , la classe 18 rassemble de très belles expositions des Ecoles Nationales professionnelles et des Ecoles pratiques de commerce et d'Industrie. Naturellement, le niveau de l'enseignement domné dans ces écoles fait que quel qu'intéressantes que soient les nombreuses machines électriques ou autres, exposées après avoir été construites dans les ateliers des écoles, ce n'est pas sous l'angle de la conception, du calcul, donc' de l'art de l'ingénieur qu'il faut juger ces petits chefs-d'cuvre, mais sous celui de la réalisation pratique, d'où la mise en lumière des qualités des professeurs, de leur ingéniosité, toutes réserves faites du reste, sur la valeur électrotechnique de leurs élèves.

L'Ecole Nationale de Vierzon, celle de Voiron, celle de Cluse, l'Ecole pratique de Grenoble (Ecole Vaucanson), celles de Denain, de Saint-Etienne, de Rives-de-Gier, de Tourcoing, de Dijon, de Marseille, de Romans, de Pont-de-Beauvoisin et de Vienne, ont véritablement fait un très bel effort. Citons, en terminant, l'exposition de l'Association post-scolaire de Grenoble, œuvre éminemment louable, groupant, le soir venu, des centaines et des centaines d'étudiants, praticiens bénévoles, et qui, contrairement à ce qui se passe dans de trop nombreuses associations analogues qui ne sont que des sociétés d'admiration mutuelle, a fourni d'admirables résultats. Un chiffre : de 700 sociétaires en 1913, l'Association post-scolaire de Grenoble a passé à 2.432 en 1925 . Les 34 cours de 1913 sont devenus les 251 de 1925 . Tel est le bilan moral de cette belle organisation d'intérêt à la fois social et national.

(S. V.). 